\title{
Assessment of diet and lifestyle factors associated with non-alcoholic fatty liver disease in a UK paediatric population
}

Non Alcoholic Fatty Liver Disease (NAFLD) is the most common cause of chronic liver disease in Western children, thought to affect up to $10 \%^{(1)}$ of the general paediatric population and up to $80 \%$ of overweight children ${ }^{(2)}$. This is the first UK based study, which aims to characterise the habitual diet, and activity behaviours of children with biopsy-confirmed NAFLD compared to BMI matched controls (obese controls, OC) to inform the development of effective prevention and management strategies.

Cases and OC were recruited from the paediatric liver unit and specialist obesity clinic respectively at King's College Hospital London, UK. On receipt of consent, children were asked to complete a 7-day Food and Activity Diary (with 7 day pedometer readings), a 24-hour Dietary Recall, a Physical Activity Questionnaire and the Dutch Eating Behaviour Questionnaire (DEBQ). The study was conducted according to the Helsinki Declaration.

Thirty-eight NAFLD and 12 OC have been recruited to date and dietary data is available for 29 (21 NAFLD; 8 OC). The groups are well matched for age (NAFLD: 13.1[SD2.2], OC: $12 \cdot 8$ [3.7] years), gender and weight, with the majority of both groups being above the 99.6 th centile for BMI. The NAFLD group were predominantly Caucasian $(83 \%)$ whereas the OC group were predominantly Black $(75 \%)(\mathrm{p}<0.001)$. NAFLD children scored non-significantly higher on all sub scales of the DEBQ, representing more negative eating behaviours. NAFLD patients were had significantly higher intakes of saturated fat and iron as assessed by $24 \mathrm{hr}$ recall when expressed as a percentage of their DRVs $(\mathrm{p}<0.05$ and $\mathrm{p}=0.02$ respectively, table 1). Interestingly, the 7-day diary records demonstrated significantly lower absolute vitamin $\mathrm{D}$ intakes in children with NAFLD $(1 \cdot 875 \mu \mathrm{g}$ versus, $3 \cdot 785 \mu \mathrm{g} p=$ 0.05). NAFLD children took a greater number of steps per day in comparison to OC (8414 [2843] versus 5965 [2066], $\mathrm{p}=0.023)$.

Table 1

\begin{tabular}{|c|c|c|c|c|c|c|}
\hline & 24 Hour R & & & 7 Day Foo & & \\
\hline Nutrient $(\%)$ & NAFLD & $\mathrm{OC}$ & $P$ value & NAFLD & $\mathrm{OC}$ & $P$ value \\
\hline Energy & $81 \cdot 6[26 \cdot 8]$ & $66 \cdot 6[22 \cdot 8]$ & 0.067 & $92 \cdot 6[26 \cdot 7]$ & $91 \cdot 8[25 \cdot 1]$ & 0.944 \\
\hline Fat & $84 \cdot 5[41 \cdot 4]$ & $56 \cdot 8[17 \cdot 9]$ & 0.031 & $89.8[68.6]$ & $92 \cdot 4[29 \cdot 0]$ & $0 \cdot 854$ \\
\hline SFA & $110 \cdot 3[57 \cdot 8]$ & $59 \cdot 0[25 \cdot 0]$ & 0.007 & $106 \cdot 6[49 \cdot 0]$ & $110 \cdot 8[37 \cdot 0]$ & 0.950 \\
\hline Protein & $159 \cdot 4[63 \cdot 5]$ & $113 \cdot 2[46 \cdot 4]$ & 0.021 & $183 \cdot 4[68.6]$ & $194 \cdot 3[64 \cdot 9]$ & 0.714 \\
\hline Iron & $86 \cdot 1[69 \cdot 4]$ & $62 \cdot 2[20 \cdot 7]$ & $0 \cdot 247$ & $86 \cdot 3[38 \cdot 4]$ & $74 \cdot 4[16 \cdot 8]$ & 0.417 \\
\hline Zinc & $97 \cdot 8[70 \cdot 8]$ & $93 \cdot 6[53 \cdot 0]$ & 0.691 & $99 \cdot 8[36 \cdot 5]$ & $93 \cdot 4[21 \cdot 3]$ & 0.652 \\
\hline Vitamin D* & $2 \cdot 0[2 \cdot 0]$ & $1 \cdot 8[1 \cdot 7]$ & 0.742 & $2 \cdot 1[1 \cdot 4]$ & $3 \cdot 7[0 \cdot 9]$ & 0.050 \\
\hline Fructose* & $14 \cdot 6[3 \cdot 3]$ & $22 \cdot 8[28 \cdot 6]$ & $0 \cdot 289$ & $17 \cdot 2[13 \cdot 0]$ & $15 \cdot 2[5 \cdot 8]$ & 0.687 \\
\hline
\end{tabular}

Data presented as \%DRV [SD] * denotes no DRV available and absolute values expressed.

This is the first study to report on the diet and activity behaviours of a UK paediatric NAFLD population. The development of NAFLD-specific lifestyle guidelines are not currently supported however this study confirms that the paediatric population are failing to conform to standard recommendations for diet or activity, which should therefore remain the focus of clinical management to optimise health and minimise disease progression.

1. Takahashi et al. (2010) World Journal of Gastroenterology 16, 5280-5285

2. Giorgio et al. (2013) BMC Pediatrics 13, 40-49 